\title{
Illuminating the deleterious effects of light at night
}

\author{
Laura K. Fonken* and Randy J. Nelson
}

Address: Department of Neuroscience and The Institute for Behavioral Medicine Research, The Ohio State University, Columbus, OH 43210, USA

* Corresponding author: Laura K. Fonken (fonken.1@osu.edu)

FI000 Medicine Reports 2011, 3:18 (doi:10.3410/M3-18)

This is an open-access article distributed under the terms of the Creative Commons Attribution-Non Commercial License

(http://creativecommons.org/licenses/by-nc/3.0/legalcode), which permits unrestricted use, distribution, and reproduction in any medium, provided the original work is properly cited. You may not use this work for commercial purposes.

The electronic version of this article is the complete one and can be found at: http://f1000.com/reports/m/3/l 8

\begin{abstract}
Technological advances, while providing many benefits, often create circumstances that differ from the conditions in which we evolved. With the wide-spread adoption of electrical lighting during the $20^{\text {th }}$ century, humans became exposed to bright and unnatural light at night for the first time in their evolutionary history. Electrical lighting has led to the wide-scale practice of 24-hour shift-work and has meant that what were once just "daytime" activities now run throughout the night; in many ways Western society now functions on a 24-hour schedule. Recent research suggests that this gain in freedom to function throughout the night may also come with significant repercussions. Disruption of our naturally evolved light and dark cycles can result in a wide range of physiological and behavioral changes with potentially serious medical implications. In this article we will discuss several mechanisms through which light at night may exert its effects on cancer, mood, and obesity, as well as potential ways to ameliorate the impact of light at night.
\end{abstract}

\section{Introduction}

At different times during our respective childhoods, we both toured Carlsbad Caverns in New Mexico. As part of the tour, the lights in the cave were turned off. The darkness was incredible, engulfing absolutely everything. In our society, we rarely experience such profound darkness; every night, our homes, work places, and streets are brightly illuminated by the glow of electric lights. We will of course always be naturally illuminated by the stars and the moon on a clear night, but we can safely say that most of the light we experience at night is unnatural.

Humans are diurnal, that is, we evolved to be active during the day and to sleep at night. As such, we never developed the ability to see well in the dark. Over time, we have, however, developed the desire to do more and more during the time we are awake, be it night or day, and so we have created an environment that corrects for our relative night blindness. Since the advent of electrical lighting around the turn of the $20^{\text {th }}$ century, humans have become increasingly exposed to bright and unnatural light at night. Urban development has further exacerbated the issue, with lighting from infrastructure straying into the atmosphere nightly (Figure 1). Today, $99 \%$ of the population in the United States and Europe, and $62 \%$ of the world's remaining population, are exposed to this "light pollution" [1]. There is no denying that the invention of electrical lighting was a boon for developing industry and technology, allowing the extension of the workday into the night and boosting economic development. However, the use of light at night continues to rapidly increase (by $6 \%$ per year) [1] without thorough (or any) consideration of its biological implications.

Light at night may appear to be an innocuous environmental manipulation, which may be why it was overlooked as a significant disease risk factor for many years; however, because of the profound impact light has on the circadian system and physiological functions such as hormone secretion, it is reasonable to conclude that exposure to light at night likely exerts a significant effect on many physiological processes. In fact, it is now evident that exposure to light at night has marked social, ecological, behavioral, and health consequences. In this 
Figure I. Satellite image of modern night-time artificial light exposure

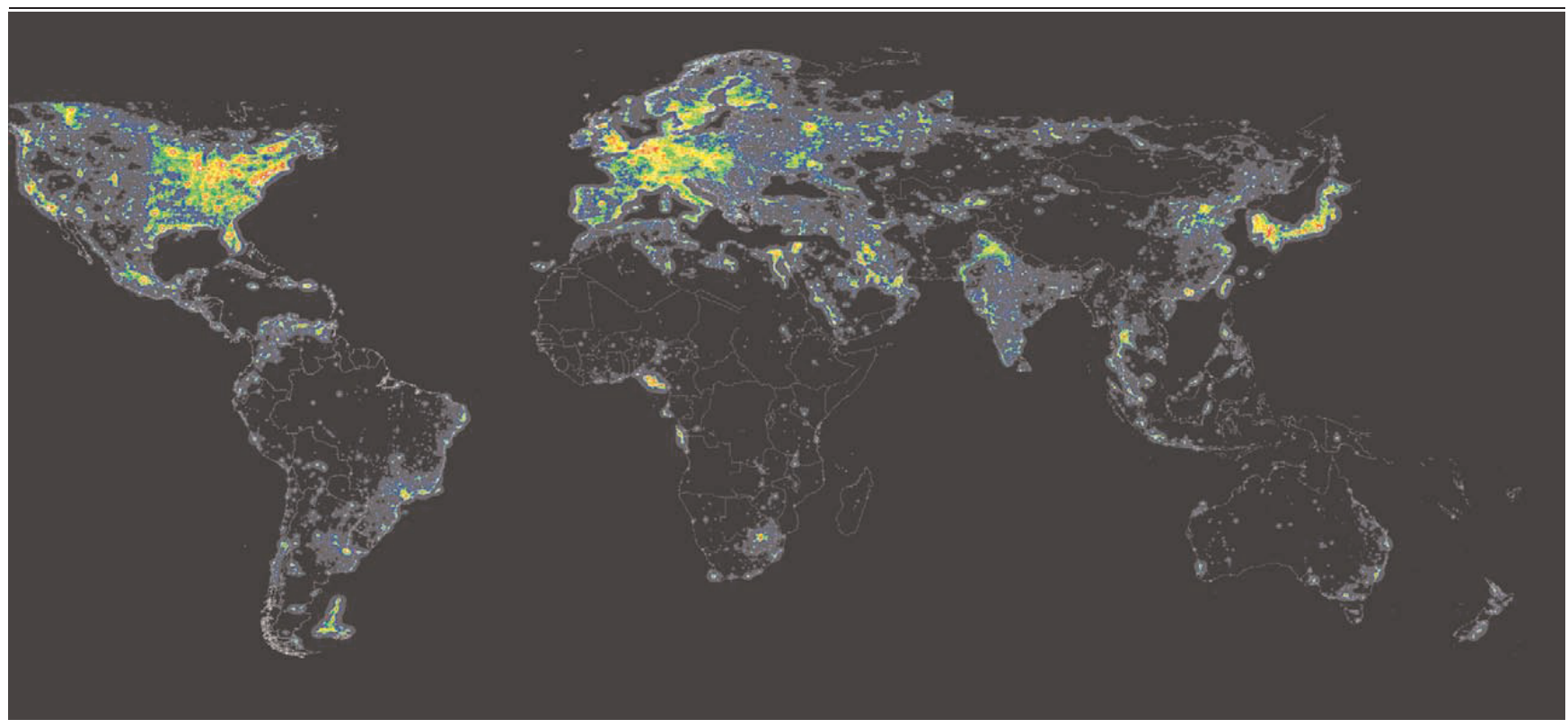

Map courtesy of Istituto di Scienza e Tecnologia dell'Inquinamento Luminoso (see [19]). (C) Royal Astronomical Society. Reproduced from the Monthly Notices of the RAS with permission from Blackwell Science.

report we will address the growing body of research on the maladaptive impact of light at night, with a specific focus on the physiological and psychological health consequences.

\section{Light at night and cancer}

The detrimental effects of light at night were first explored by epidemiological studies investigating cancer risk in shift workers. It began in the late 1970s, when it was proposed that the pineal gland (which produces the hormone melatonin, a modulator of sleep/wake patterns) influenced the development of breast cancer [2]. However, it was not until the late 1980s, when Richard Stevens postulated that night-time light exposure may represent a risk factor for breast cancer development in Westernized societies, that research in this area began in earnest [3]. Fast-forward to today, and there is now strong epidemiological and experimental work in support of the hypothesis that light at night impacts breast cancer risk and development, which will be discussed below. Indeed, the International Agency on Research on Cancer has classified shift work as "probably carcinogenic to humans" and some countries (e.g., Denmark) have classed breast cancer as an occupational health risk associated with shift work. More recent studies have also begun to look at the association between exposure to light at night in general (outside the context of shift work) and risk for developing certain types of cancer such as breast and prostate cancer.
Light exposure during the night may increase breast cancer risk via several mechanisms. First, light at night affects certain cancers by decreasing levels of the pineal hormone melatonin, which is suppressed when an individual is exposed to light at night. Melatonin is produced and released primarily during the night, with a longer night corresponding to an increased duration of melatonin production, and is important for coordinating endogenous circadian timing. Melatonin production is disrupted by light at night, with bright light intensities suppressing melatonin secretion for the entire night. Melatonin may influence breast cancer development via two primary routes: (a) a decrease in melatonin may increase estrogen/progesterone signaling, two known breast cancer promoters; and/or (b) melatonin may act directly on cancer and cancer-fighting cells that have melatonin receptors, reducing tumor growth. Melatonin can also affect tumor onset and development via its actions on oxidative stressors [4].

Despite the fact that the association between light at night and breast cancer has been known for several decades, appropriate animal models to study light exposure at night, and in vitro work to provide direct causational evidence, have been slow to develop. Blask et al. made pathbreaking advances in light at night and cancer research in 2005 by providing direct evidence of the influence of light at night on breast cancer xenografts in nude rats [5]. Rats bearing human breast cancer 
xenografts or rat hepatomas were exposed to increasing white fluorescent light intensities during their 12-hour dark phase, which led to a dose-dependent suppression in melatonin. As light intensities increased, tumor growth rates increased and latency to tumor onset decreased. This dose response of cancer cells to different intensities of light at night demonstrates how even low level nocturnal lighting can affect cancer development. Melatonin receptors are generally found in the brain and some peripheral tissues; however, it was postulated that the changes in tumor growth and onset seen here may be a consequence of melatonin receptors directly embedded on the tumor cells. mRNA for melatonin 1 and 2 receptors ( $\mathrm{MT}_{1}$ and $\mathrm{MT}_{2}$, respectively) was characterized on the human breast cancer xenografts, providing support that both of these melatonin receptors are expressed in solid tumors in vivo, meaning that melatonin has the ability to directly affect cancer cells. Importantly, no increase in either serum corticosterone or estradiol levels (adrenocortical/ovarian hormones known to influence breast cancer risk) during the dark was observed, meaning melatonin likely worked directly on tumor cells, and not via hormones, to modulate cancer growth.

In the same study, human breast cancer xenografts were perfused in situ with blood collected from premenopausal female volunteers either during the day, during the night under darkness, or during the night following exposure to white light. Melatonin-rich blood collected during the dark suppressed tumor growth signaling activity when it was perfused through xenografts, whereas neither the daytime nor night-time light exposure blood samples (both melatonin-deficient) suppressed tumor-mediated signaling, providing further evidence for the role of melatonin in the incidence and growth of breast cancer associated with exposure to light at night.

With the invention of electric lights, daylight hours have gradually been extended 4-7 hours depending on the season. As a result, overall melatonin production is suppressed in most people, not just shift workers. This marked decrease likely reduces the oncostatic effects of melatonin, which has led researchers to hypothesize that light at night may contribute to the increased incidence of tumors in the general population. To determine whether general lighting levels outside of a shift-working population also relate to breast cancer incidence, Kloog et al. obtained the average night-time illumination levels from 164 countries using the US Defense Meteorological Satellite Program, and concurrently evaluated breast cancer incidence from the GLOBOCAN 2002 database [6]. Geographic information systems were then used to match the cancer rates with light at night levels obtained from the satellite imaging and other relevant socioeconomic data (that could also be important in explaining variations in cancer rates). A strong correlation was revealed between breast cancer rates and light at night. This is an important addition to the growing body of research on light at night and breast cancer because it demonstrates a population-level trend, whereas previous analyses examined only specific groups. Women living in the darkest communities may have prolonged durations of nightly melatonin, lowering their risk of breast cancer.

Understanding the impact of light on our circadian clock-the internal oscillator that controls our diurnal rhythms by using transcriptional-translational feedback loops involving several clock genes-is critical to fully understanding the link between light at night and cancer. This is because circadian clock genes are important regulators of cell proliferation and apoptosis; therefore, disturbances of clock function may disrupt cellular processes that may lead to cancer. The circadian clock is most potently entrained by light, so when individuals travel to different time zones or stay out late on the weekends they disrupt their internal timing. Recent work in circadian clock gene-mutant mice has supported the hypothesis that disruption of the circadian clock leads to increased tumor development [7]. Furthermore, mimicking "jet-lag" in these mice by shifting their light cycle accelerates tumor development. Taken together, these studies demonstrate that unnatural light at night can affect tumor development and growth via several mechanisms.

\section{Light at night and mood}

The prevalence of major depression has also increased in recent decades. Although recognized factors such as better diagnoses, changing diagnostic criteria, and dietary changes account for some of the rise in depression rates, additional variables are likely involved. Depression represents an interaction between genes and the environment, and the increased rates of depression are occurring too quickly to be attributable to changes in genes only. Thus, it seems reasonable to hypothesize that recent environmental changes may partially account for the increasing incidence of depression. Light at night is one likely environmental candidate, although there are several others.

Fluctuations in daylight from season to season, abnormalities in the circadian clock, sleep disorders, and shift work are associated with depression in some subpopulations. At the opposite end of the spectrum to exposure to too much light (at night), depression is often considered in the context of individuals who experience too little 
lighting (during the day). For example, symptoms of seasonal affective disorder (SAD), a mood disorder in which depressive symptoms are restricted to a particular time of year (primarily the winter when day lengths are short), are often ameliorated by light therapy. In SAD, it is hypothesized that circadian rhythms are misaligned and melatonin secretion remains high in the morning. Thus, morning exposure to bright light truncates melatonin secretion. The susceptibility of some individuals to changes in day length led us to hypothesize that humans may also be sensitive to dramatic increases in environmental lighting. It stands to reason that a decrease in light during 'normal' daylight hours (SAD) and an increase in light during 'normal' night-time hours causes a similar effect because adaptive processes have optimized the physiological and behavioral regulation of individuals according to changing day lengths and circadian cycles, therefore sudden, prolonged or artificial changes in light cycles will likely affect mood and behavior.

We originally became interested in how light at night interacts with mood when a graduate student in our lab was hospitalized with a Staphylococcus infection. One of his primary complaints while staying in the hospital was the high levels of light during the night. Because elevated rates of depression are common among in-patient populations, we were curious whether light at night may be a contributing factor. In our first study on light at night and depression we worked with male mice that were housed in either a normal light/dark cycle or in constant light [8]. Additionally, mice either had access to an opaque tube, in which they could escape light, or a clear tube that provided no mechanism for light escape. After living in these two different conditions for 3 weeks, we tested the mice for depressive-like behaviors in a swim test and a sugar water test.

One key symptom in human depression is anhedonia, which is a failure to engage in activities that the individual previously deemed pleasurable. Mice that were housed in constant light drank less sugar water than those in the normal dark/light phase, indicating an anhedonic response. Furthermore, mice housed in constant light with a clear tube showed more depressive-like behavioral responses in the swim test. Mice housed in constant light with an opaque tube did not display an increase in depressive behaviors in the swim test, suggesting that ability to escape the light by entering the opaque tube partially reversed the depressive responses.

One key issue with extrapolating our initial study on light at night and depression in male mice to humans is that humans are generally not exposed to continuous bright lighting throughout the day and night like the mice in the investigation were. Those mice had no temporal cues to establish time of day, and their circadian rhythms were likely free running. Furthermore, depression is almost twofold higher among women as compared with men [9]. For these reasons, we conducted a follow-up study in which we evaluated the impact of dim light at night on depression in female Siberian hamsters [10]. Hamsters were either housed in a standard light/dark cycle or a light/dim light cycle in which they experienced dim light (5 lux) during the dark phase. Similar to male mice, hamsters chronically exposed to dim light at night were more immobile in the swim test (an indication of behavioral despair) and consumed less sucrose solution. Taken together, these behavioral responses indicate a depression-like state. Depression is associated with hippocampal structural changes, which led us to evaluate neuronal morphology. Hamsters exposed to dim light at night had reduced spine density in the hippocampus, which has been associated with depression in other animal models. Overall, light at night may alter hippocampal structure leading to behavioral changes.

It is possible that light at night contributes to depression via suppressed melatonin. For example, agomelatine, a melatonin-receptor agonist that also antagonizes serotonin $\left(5-\mathrm{HT}_{2 \mathrm{c}}\right)$ receptors, is an effective antidepressant. However, the male mice used in our initial study do not produce melatonin, which suggests that melatonin is not the primary mechanism driving the changes in mood. Overall, we hypothesize that disruption of the circadian system may in part lead to differences seen in mood. Another study, which looked at the behavior of clockgene-mutant mice, demonstrated how disruption of the circadian system can influence mood in general. The mice in this study displayed many symptoms described in human mania (often experienced cyclically with depression in illnesses such as bipolar disorder). Symptoms included hyperactivity, decreased sleep, lowered depression-like behavior, lowered anxiety, and increased motivation for rewarding stimuli such as sugar and cocaine [11]. However, it is important to note that animals exposed to dim light at night do not change activity patterns, so circadian disruption is more likely occurring at the cellular level.

\section{Light at night and body mass}

We first discovered that light at night impacted metabolism serendipitously. While conducting research on light at night and mood we noticed a strange trend; mice exposed to light at night were becoming fat. Because of the rapid increase in obesity paralleling increasing light levels and the association between shift work and elevated body mass index (BMI) $[12,13]$, we further 
explored this phenomenon by examining the effects of constant light and dim light at night on metabolic parameters in mice. Both groups of mice exposed to light at night (either dim or constant bright) had an increase in body mass throughout our initial study compared with mice housed in a standard light/dark cycle, despite equivalent levels of food intake and total daily activity [14] (Figure 2). Furthermore, after 4 weeks in their respective light conditions, light-at-night mice displayed impaired glucose tolerance, which is indicative of a prediabetic-like state. Although total daily food intake and activity were not disrupted, light-at-night mice ate more food during their rest phase than mice housed in standard light cycle did in their rest phase. (For both sets of mice, the rest phase was the daylight phase, as the mice are nocturnal.) Furthermore, correlation analyses confirmed that percentage of food consumed during the day was positively related to increased body mass. In a follow-up study, restricting food availability to a more typical time of feeding in light-at-night mice prevented weight gain, further implicating timing of food intake in body mass gain.

Light at night may also influence metabolism by suppressing melatonin; however, the influence of melatonin on our results are unclear because the mouse strain under study produce very low levels of melatonin. Much as with our work on light at night and depression, we believe that the changes in metabolism may be due in part to disruption of the circadian system. Metabolism and the circadian clock are intrinsically related, with desynchrony of activity and food intake causing metabolic adjustments. For example, building on previous research, Joseph Bass and colleagues recently demonstrated the importance of functional cross-talk among clock genes for maintaining energy homeostasis [15]. Clock-gene-mutant mice have reduced insulin production and impaired glucose tolerance. Furthermore, clock mutations have previously been

Figure 2. Exposure to light at night increases body mass in mice

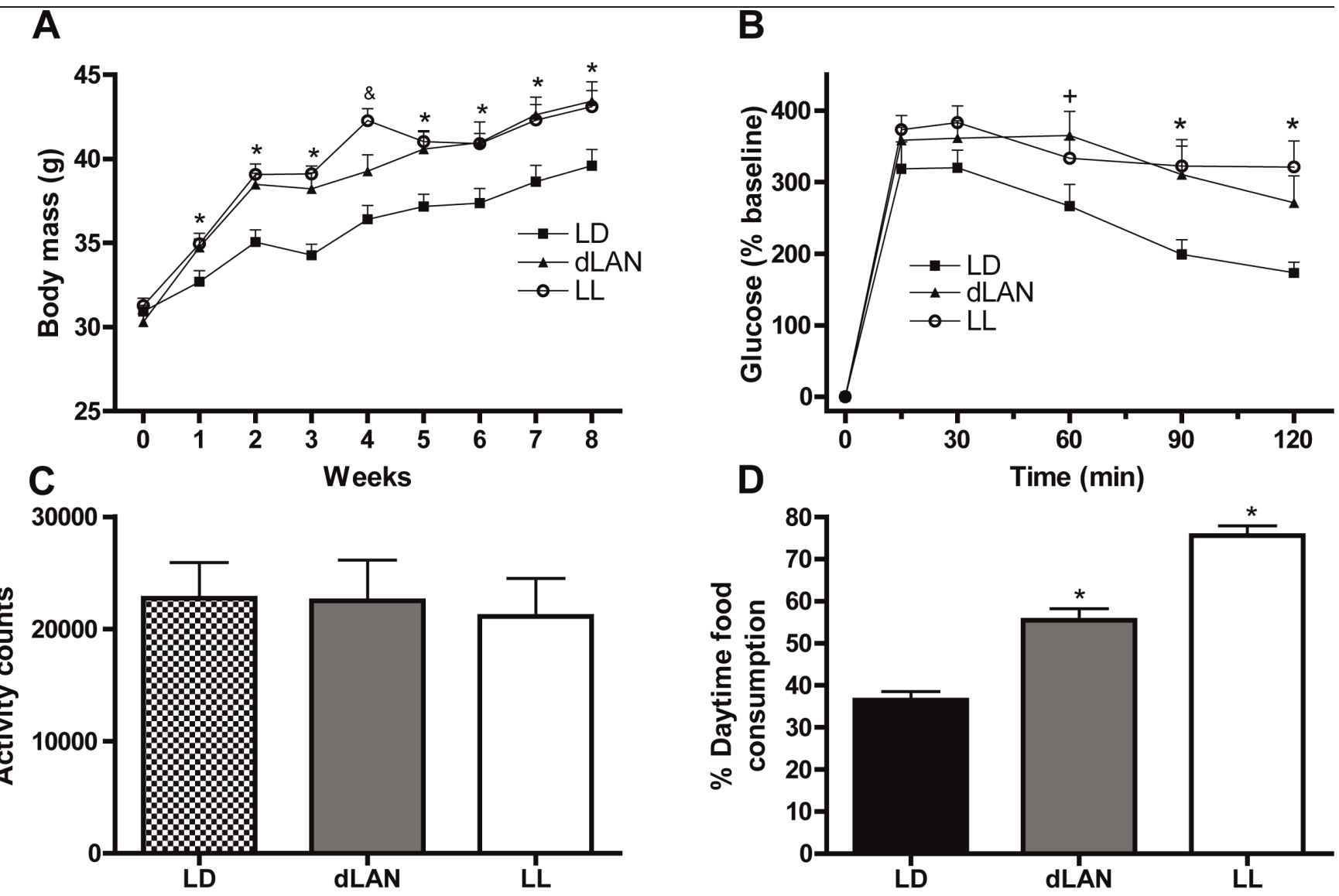

(A) Mice exposed to either dim light at night (dLAN) or constant light (LL) had increased weight gain relative to mice in a standard light/dark cycle (LD). dLAN and LL mice also reduced glucose tolerance (see (B)) despite similar levels of activity (see (C)) and total daily food consumption (not shown). However, mice exposed to LAN consumed significantly more food during the light phase (when mice would generally be resting) than those in a standard light/dark cycle (see (D)). Adapted from Fonken et al., 2010 [14]. 
shown to result in obesity [16]. Because of the multiple commonalities in phenotype between mice exposed to light at night and those with disruption in circadianrelated genes, one may hypothesize that light at night causes changes in metabolism through dysregulation of peripheral clock genes.

Recent work with human adipose tissue further supports the role of clock-gene regulation in body mass maintenance and metabolism [17]. Gómez-Abellán et al. [17] showed that human adipose tissue expresses core molecular components of the circadian system. Importantly, the researchers also demonstrated that expression of one of these critical clock genes, Per2, was inversely related to waist circumference (which the investigators used as an indication of "metabolic syndrome"). These results may begin to formulate a mechanism for the association between increased BMI and working night shifts; shift workers often experience significant circadian disruption, which in turn may result in changes in internal clock coordination impacting adipose tissue.

\section{Conclusion}

A common risk factor in many of the pathologies associated with exposure to light at night is a change in immune function, notably inflammatory responses, and recent research has demonstrated that light at night may detrimentally affect the immune system [18]. Thus, in addition to investigating the influence of melatonin and circadian disruption as mechanisms contributing to the maladaptive affects of light at night, characterization of the inflammatory response is also warranted.

One important population that is often neglected when considering light at night is patients in hospitals. While multiple epidemiological studies have been conducted on nurses, there are no studies on the impact of light at night on the patients with whom they work. Many in-patients are already at high risk of increased inflammation and disrupted physiology, which may be exacerbated by light at night.

Preventing the general population from excessive exposure to light at night can be achieved with relatively low-cost manipulations, such as using curtains to block out street lights, turning off hallway lights, and removing all light sources, including televisions and computers, from bedrooms. However, these methods do not prevent the extension of daytime hours that many of us experience, but by no means do we recommend that everyone go to bed at sunset. Rather, it may be important for people to try to keep a consistent schedule and avoid rapid shifts in their waking hours. This is often unavoidable in shiftworking populations, and there are ongoing studies currently comparing visual aids that may alleviate some of the maladaptive effects of exposure to light at night in shift workers. More specifically, not all lighting has an equal effect; the intrinsically photosensitive retinal ganglion cells that project to the master circadian clock in the brain contain melanopsin and are most responsive to the blue region of the visible spectrum (ranging from 450 to $485 \mathrm{~nm}$ ), with longer wavelengths of lighting minimally impacting the circadian system. Manipulation of wavelength may prove effective in blocking out some of the light-induced physiological changes. Current research investigating the effectiveness of goggles designed to block out blue wavelength lighting on preventing light-induced melatonin suppression is ongoing. Furthermore, work environments could potentially use lighting sources that emit less blue light, which unfortunately is at odds with the push for energy-saving compact fluorescent bulbs.

Modern society now functions on a 24-hour schedule. Although there are many economic and other societal benefits to such a schedule, there is converging evidence from epidemiological and experimental work that light at night has unintended, maladaptive consequences. In many ways, this field of study is just beginning; further characterization of the impact of light at night is needed along with effective interventions to ameliorate the unintended negative effects of light at night on health.

\section{Abbreviations}

BMI, body mass index; $\mathrm{M}_{1}$ and $\mathrm{M}_{2}$, melatonin receptor 1 and 2.

\section{Competing interests}

The authors declare that they have no competing interests.

\section{Acknowledgements}

The authors thank Zachary M. Weil for his helpful comments on the manuscript.

\section{References}

I. Navara KJ, Nelson RJ: The dark side of light at night: physiological, epidemiological, and ecological consequences. J Pineal Res 2007, 43:215-24.

2. Cohen M, Lippman M, Chabner B: Pineal gland and breast cancer. Lancet 1978, 2:138I-2.

3. Stevens RG: Electric power use and breast cancer: a hypothesis. Am J Epidemiol 1987, I 25:556-6I.

FI000 Factor 6

Evaluated by Randy Nelson 19 Aug 201I

4. Karbownik M, Reiter RJ: Melatonin protects against oxidative stress caused by delta-aminolevulinic acid: implications for cancer reduction. Cancer Invest 2002, 20:276-86.

5. Blask DE, Brainard GC, Dauchy RT, Hanifin JP, Davidson LK, Krause JA, Sauer LA, Rivera-Bermudez MA, Dubocovich ML, Jasser SA, Lynch DT, Rollag MD, Zalatan F: Melatonin-depleted 
blood from premenopausal women exposed to light at night stimulates growth of human breast cancer xenografts in nude rats. Cancer Res 2005, 65: III 174-84.

FI000 Factor 6

Evaluated by Randy Nelson 19 Aug 2011

6. Kloog I, Stevens RG, Haim A, Portnov BA: Nighttime light level co-distributes with breast cancer incidence worldwide. Cancer Causes Control 2010, $21: 2059-68$.

FI000 Factor 6

Evaluated by Randy Nelson 19 Aug 2011

7. Lee S, Donehower LA, Herron AJ, Moore DD, Fu LN: Disrupting Circadian Homeostasis of Sympathetic Signaling Promotes Tumor Development in Mice. PloS One 2010, 5:el0995.

FI000 Factor II

Evaluated by Mary Harrington 18 Jun 2010, Robin McAllen 02 Aug 2010, Randy Nelson 19 Aug 2011

8. Fonken LK, Finy MS, Walton JC, Weil ZM, Workman JL, Ross J, Nelson RJ: Influence of light at night on murine anxiety- and depressive-like responses. Behav Brain Res 2009, 205:349-54.

9. Kessler RC, McGonagle KA, Swartz M, Blazer DG, Nelson CB: Sex and depression in the National Comorbidity Survey. I: Lifetime prevalence, chronicity and recurrence. J Affect Disord 1993, 29:85-96

10. Bedrosian TA, Fonken LK, Walton JC, Haim A, Nelson RJ: Dim light at night provokes depression-like behaviors and reduces CAI dendritic spine density in female hamsters. Psychoneuroendocrinology 201I, 36: 1062-9.

II. Roybal K, Theobold D, Graham A, DiNieri JA, Russo SJ, Krishnan V, Chakravarty S, Peevey J, Oehrlein N, Birnbaum S, Vitaterna MH, Orsulak P, Takahashi JS, Nestler EJ, Carlezon WA Jr, McClung CA: Mania-like behavior induced by disruption of CLOCK. Proc Natl Acad Sci U S A 2007, 104:6406-II.

FI000 Factor 7

Evaluated by Ralf Jockers 18 Apr 2007, Randy Nelson 19 Aug 2011

12. van Amelsvoort LG, Schouten EG, Kok FJ: Duration of shiftwork related to body mass index and waist to hip ratio. Int J Obes Relat Metab Disord 1999, 23:973-8.
13. Parkes KR: Shift work and age as interactive predictors of body mass index among offshore workers. Scand J Work Environ Health 2002, 28:64-7I.

14. Fonken LK, Workman JL, Walton JC, Weil ZM, Morris JS, Haim A, Nelson RJ: Light at night increases body mass by shifting the time of food intake. Proc Natl Acad Sci U S A 2010, 107:18664-9.

FI000 Factor 9

Evaluated by Michael Symonds 19 Oct 2010, Andrea Gore 27 Oct 2010

15. Marcheva B, Ramsey KM, Buhr ED, Kobayashi Y, Su H, Ko CH, Ivanova G, Omura C, Mo S, Vitaterna MH, Lopez JP, Philipson LH, Bradfield CA, Crosby SD, JeBailey L, Wang X, Takahashi JS, Bass J: Disruption of the clock components CLOCK and BMALI leads to hypoinsulinaemia and diabetes. Nature 2010, 466:627-31.

FI000 Factor 10

Evaluated by Randy Nelson 09 Aug 2010, Marc Montminy 27 Aug 2010, Michael Menaker 28 Sep 2010

16. Turek FW, Joshu C, Kohsaka A, Lin E, Ivanova G, McDearmon E, Laposky A, Losee-Olson S, Easton A, Jensen DR, Eckel RH, Takahashi JS, Bass J: Obesity and metabolic syndrome in circadian Clock mutant mice. Science 2005, 308:1043-5.

FI000 Factor 12

Evaluated by Eric Widmaier 25 Aug 2005, Louis Ptacek 28 Apr 2005

17. Gómez-Abellán P, Hernández-Morante JJ, Luján JA, Madrid JA, Garaulet M: Clock genes are implicated in the human metabolic syndrome. Int J Obes (Lond) 2008, 32:121-8.

FI000 Factor 6

Evaluated by Randy Nelson 19 Aug 2011

18. Bedrosian TA, Fonken LK, Walton JC, Nelson RJ: Chronic exposure to dim light at night suppresses immune responses in Siberian hamsters. Biol Lett 2011, 7:468-71.

19. Cinzano P, Falchi PF, Elvidge CD: The first World Atlas of the artificial night sky brightness. Mon Not $R$ Astron Soc 200I, 328:689-707. 